ORIGINAL ARTICLE

\title{
Estimation of leaf area index of Pinus taeda L. and Cupressus lusitanica Mill. by vegetation indices
}

\author{
Estimativa de índice de área foliar em Pinus taeda L. e Cupressus lusitanica Mill. \\ por índices de vegetação oriundos de dados ópticos
}

\author{
Carla Talita Pertille ${ }^{1}$ (D), Marcos Felipe Nicoletti ${ }^{1}$ (D), Larissa Regina Topanotti ${ }^{1}$ (D), Mario Dobner Júnior ${ }^{1}$ (1) \\ ${ }^{1}$ Universidade do Estado de Santa Catarina - UDESC, Lages, SC, Brasil
}

How to cite: Pertille, C. T., Nicoletti, M. F., Topanotti, L. R., \& Dobner Júnior, M. (2020). Estimation of leaf area index of Pinus taeda L. and Cupressus Iusitanica Mill. by vegetation indices. Scientia Forestalis, 48(125), e3047. https://doi.org/10.18671/scifor.v48n125.03

\begin{abstract}
This research aimed to quantify the leaf area index (LAI) of a forest of Pinus taeda L. and Cupressus Iusitanica Mill. using optical data from the Landsat-8/OLI and Sentinel-2/MSI sensors. For that, 50 circular plots of $500 \mathrm{~m}^{2}$ in the area were allocated, in which LAI readings were performed using the LAI-2200 equipment. The remotely located data comprised images of the Landsat-8/OLI and Sentinel-2/MSI orbital sensors. After digital processing, 15 vegetation indexes were calculated for each image used. These data were correlated with LAl by plot. With the best indexes correlated with the LAI variable, regression models were constructed using the Stepwise technique (Backward and Forward). The best model was determined based on the adjusted coefficient of determination ( $R^{2}$ adjusted), standard error of estimate (Syx\%), Akaike Information Criterion (AIC), Bayesian Information Criterion (BIC) and Root Mean Squared Error (RMSE). The results showed that there was a significant correlation among the average rates per plot and the LAI per plot. The fitted models developed with the indexes derived from Sentinel- 2 had superior performance to the models built with the Landsat- 8 data. The best estimate was the LAI per plot with $4.75 \%$ of error and adjusted $\mathrm{R}^{2}$ of 0.9134 . There was no significant difference between the LAl obtained from the field campaign and the LAI estimated by the spectral data (Landsat-8/OLI and Sentinel-2/MSI). However, it is recommended to test this methodology using sensors of high spatial resolution and with other species of the genera Pinus and Cupressus.
\end{abstract}

Keywords: Remote sensing; Biophysical variables; Orbital images.

\section{Resumo}

Essa pesquisa objetivou quantificar o índice de área foliar (IAF) em uma floresta de Pinus taeda L. e Cupressus lusitanica Mill. utilizando dados ópticos oriundos dos sensores Landsat-8/OLI e Sentinel-2/MSI. Para tanto, foram alocadas 50 parcelas circulares de $500 \mathrm{~m}^{2}$ na área, nas quais foi realizada uma leitura de IAF utilizando o equipamento LAI-2200. Os dados remotamente situados compreenderam imagens dos sensores orbitais Landsat-8/OLI e Sentinel-2/MSI. Após o processamento digital, foram calculados 15 índices de vegetação para cada imagem utilizada. Esses dados foram correlacionados com o IAF por parcela. Com os melhores índices correlacionados com a variável IAF, construiu-se modelos de regressão a partir da técnica de Stepwise (Backward e Forward). O melhor modelo foi determinado com base no coeficiente de determinação ajustado ( $R^{2}$ ajustado), erro padrão da estimativa (Syx\%), Critério de Informação de Akaike (AIC), Critério de Informação Bayesiano (BIC) e Raiz do Erro Médio Quadrático (RMSE). Os resultados revelaram que houve correlação significativa entre os índices médios e o IAF por parcela. Os modelos ajustados desenvolvidos com os índices derivados do Sentinel-2 tiveram desempenho superior aos modelos construídos com os dados do Landsat-8. O melhor estimou o IAF com $4,75 \%$ de erro e $\mathrm{R}^{2}$ ajustado de 0,9134 . Não houve diferença significativa entre o IAF obtido pela

Financial support: None.

Conflict of interest: Nothing to declare.

Corresponding author: carlatpertille@gmail.com

Received: 6 August 2018.

Accepted: 12 June 2019.

Editor: Francides Gomes da Silva Júnior

(c) This is an Open Access article distributed under the terms of the Creative Commons Attribution License, which permits unrestricted use,

c) distribution, and reproduction in any medium, provided the original work is properly cited. 
campanha de campo e o IAF estimado pelos dados espectrais (Landsat-8/OLI e Sentinel-2/MSI). Entretanto, recomenda-se testar essa metodologia utilizando sensores de alta resolução espacial e com outras espécies dos gêneros Pinus spp. e Cupressus spp.

Palavras-chave: Sensoriamento remoto; Variáveis biofísicas; Imagens orbitais.

\section{INTRODUCTION}

Species of the genus Pinus spp. are among the main species planted in southern Brazil. In Santa Catarina, in 2016, there were 545,835 hectares of forests of this genus, representing $34 \%$ of the plantations in the country (Indústria Brasileira de Árvores, 2017). Among this genus' species, one of the most important is Pinus taeda $\mathrm{L}$.

Another genus with great potential for planting and uses in southern Brazil comprises the genus Cupressus spp., and Cupressus lusitanica Mill. stands out, because it has the potential for wood production and it is indicated for cultivation in Santa Catarina (Shimizu et al., 2006).

Considering the relevance of these species, an important indicator to monitor the vigor and structure of the canopy of these plantations is the Leaf Area Index (LAI). Defined by Watson (1947) as the unilateral total area of leaf tissue per unit of soil surface area, this index is related to photosynthesis and transpiration and it is also considered as an indicator of the leaves' geometric structure and density. The literature addresses several concepts for LAI, defined by leaf forms (flat or needles) and methods of obtaining it (Melnikova et al., 2018). For leaves with needle geometry, the concept of Myneni et al. (1997) should be used, which defines LAl as the maximum leaf area projected per unit of soil surface.

This biophysical parameter is related to the energy and carbon exchanges between the vegetation and the atmosphere, and it is essential to gather information about the LAl in order to understand the changes in energy and carbon cycle in response to climate change (Wang et al., 2004). Besides, Landsberg \& Sands (2011) point out the LAl, as an indicator of agricultural and forestry areas productivity.

There are two methods of obtaining LAl: direct or destructive and indirect or non-destructive (Rody et al., 2014). The first involves in situ approaches using model tree techniques, litter collection, and litterfall collection (Nasahara et al., 2008; Nagai et al., 2014). The indirect ones involve both contact methods such as slope quadrature and allometry (Bolstad et al., 2001) and non-contact by optical methods, using data from Remote Sensing (SR).

SR data with high temporal resolution and large-scale observation capability allowed the emergence of several studies to predict LAI. The methodologies tested with remotely located data involved the application of statistical approaches (vegetation indices), radiative transfer models (RTM) (Dorigo et al., 2007; Baret \& Buis, 2008) and statistical methods, including simple linear regression (Broge \& Leblanc, 2001), multiple linear regression (Heiskanen, 2011) and partial least squares regression (PLSR) (Cho et al., 2007). These regression models are site-specific and sensor-specific, and their performance may be hampered by factors such as differences in surface and sun position properties, as well as geometry visualization (Verrelst et al., 2010).

Vegetation indices from passive optical data have been used in the estimation of LAI in large areas. According to Almeida et al. (2015), vegetation indices are a way to highlight vegetation spectral behavior using combinations of bands, such as red and near infrared. This makes these indexes more sensitive to changes in the canopy structure than the individual bands. There are several indexes in the literature, but the most used for the estimation of LAI are the Simple Ratio (SR) developed by Jordan (1969), the Normalized Difference Vegetation Index (NDVI) (Rouse et al., 1974) and the Soil Adjusted Vegetation Index (SAVI) (Huete, 1988).

Majasalmi et al. (2013) state that the quantification accuracy of LAI by VIs depends on the following factors: tree species, forest structure and moment of measurement. As a result, Sasai et al. (2011) recommend using remote sensors with spatial resolution less than or equal to 30 meters, in order to obtain accurate reflectance values. In this sense, the Landsat-8/OLI sensor is indicated for these surveys, since it has the best estimates of reflectance, spatial resolution of 30 meters and the presence of a narrower spectral band of the near infrared band (Roy et al., 2014). Another recommended sensor refers to the Sentinel-2 satellite mission 
based on 10-meter spatial resolution, 5-day time resolution, and free data availability. In the forest area, studies with Sentinel-2 are still insufficient; however, in the agricultural area, Clevers et al. (2017) have already investigated the potential of this sensor for LAI prediction.

In this scenario, the objective of this study was to quantify the leaf area index in a forest of Pinus taeda L. and Cupressus lusitanica Mill. using optical data from the Landsat-8/OLI and Sentinel-2/MSI sensors.

\section{MATERIAL AND METHODS}

\section{Study area}

The research was carried out in a multilayer system of 36-year-old Pinus taeda L. and 13-year-old Cupressus lusitanica Mill. located in the municipality of Campo Belo do Sul, in the highlands of Santa Catarina (Figure 1). The area has a mean altitude of 1,017 m.a.s.l. and the climate, according to the classification of Koppen, is classified as Cfb (subtropical), with an average temperature of $15.7^{\circ} \mathrm{C}$ and annual rainfall is 1,647 mm (Alvares et al., 2013).

c)

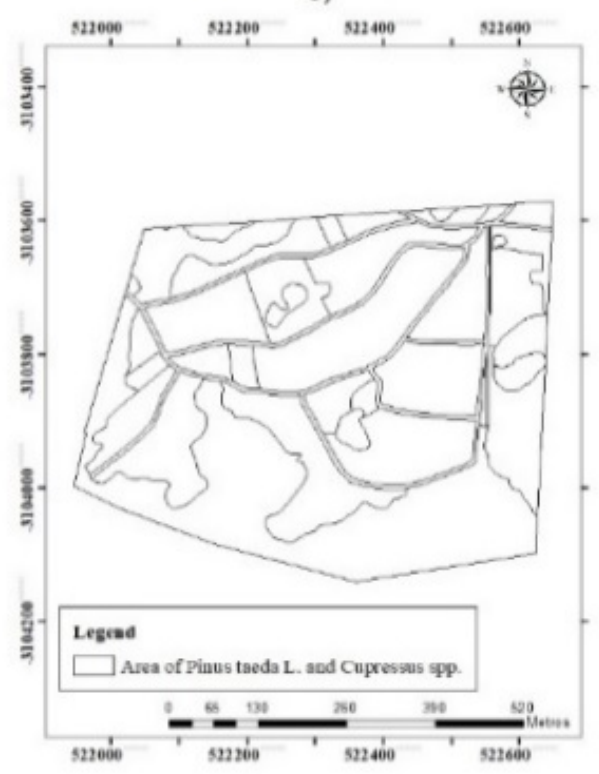

A)

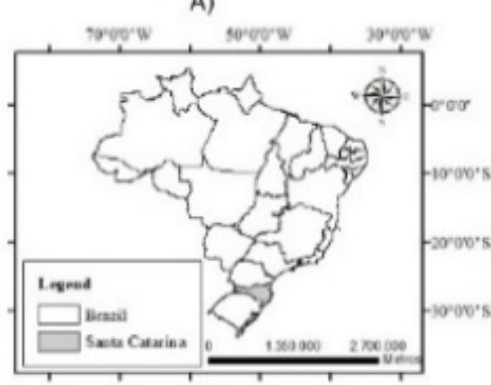

B)

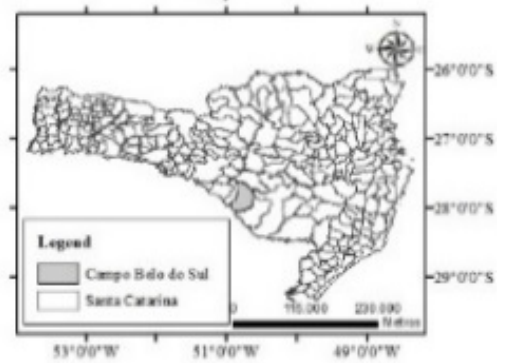

Figure 1. Location of the study area A) Brazil, B) Santa Catarina and C) forest of Pinus taeda L. and Cupressus lusitanica Mill.

\section{LAI Measurements}

Concerning field measurements, 10 circular plots of $500 \mathrm{~m}^{2}$ were allocated. The central coordinate of each plot was obtained using a receptor Garmin Etrex GPS, where the data were geo-referenced in the reference system World Geodetic System 1984 (WGS-84). Subsequently, these data were converted to the Geocentric Reference System of the Americas - SIRGAS 2000 with Mercator Transverse Universal Projection System (UTM) Fuso $22 \mathrm{~S}$.

In each plot, the LAl was collected with the LAI-2200 equipment in April 2018. The equipment calibration was performed in an obstacle-free area and then within the forest. The value of the LAI per plot corresponded to the average of five consecutive readings in the following directions: center, north, south, east, and west. Thus, 50 LAl values were obtained (five values for each plot). 


\section{Remotely located data}

The Landsat- 8 satellites were used with the OLI (Operational Land Imager) sensor and Sentinel-2 with the MSI (Multispectral Instrument) sensor. The characteristics of these sensors are described in United States Geological Survey (2013) and European Space Agency (2010).

The criteria for obtaining the images of the sensors were close dates to the field activities and absence or low cloud cover.

The Landsat-8/OLI sensor image was acquired from the United States Geological Survey platform, dated 04/22/2018. The Sentinel-2/MSI image was obtained from the Copernicus Open Access Hub portal for the same date. The orbit 221 and point 79 was used for both images.

The images were processed in the ENVI (Environment for Visualizing Images) computational application, using the FLAASH (Fast Line-of-sight Atmospheric Analysis of Hypercubes) algorithm for atmospheric correction. The Sentinel-2/MSI sensor image was re-scanned for 10 meters in the Sentinel Application Platform (SNAP).

With the correct digital processing performed on the images, the vegetation indices described in Table 1 were calculated:

Table 1. Vegetation Indices calculated for the study area.

\begin{tabular}{|c|c|c|}
\hline VI & Formula & Reference \\
\hline ARVI & $\frac{\rho \mathrm{NIR}-2(\rho \mathrm{RED}-\rho \mathrm{BLUE})}{\rho \mathrm{NIR}+2(\rho \mathrm{RED}-\rho \mathrm{BLUE})}$ & $\begin{array}{c}\text { Kaufman \& Tanré } \\
\text { (1992) }\end{array}$ \\
\hline$C L_{\text {green }}$ & $\frac{\rho \text { NIR }}{\rho \text { GREEN }-1}$ & $\begin{array}{l}\text { Gitelson et al. (2003a, } \\
\text { 2003b) }\end{array}$ \\
\hline DVI & $\gamma \rho \mathrm{NIR}-\rho \mathrm{RED}$ & $\begin{array}{c}\text { Richardson \& } \\
\text { Wegand (1977) }\end{array}$ \\
\hline EVI & $2.5^{*} \frac{(\rho \mathrm{NIR}-\rho \mathrm{RED})}{\rho \mathrm{NIR}+\left(6^{*} \rho \mathrm{RED}-7.5^{*} \rho \mathrm{BLUE}\right)+1}$ & Huete et al. (1997) \\
\hline $\mathrm{EVI}_{2}$ & $2.5^{*} \frac{(\rho \mathrm{NIR}-\rho \mathrm{RED})}{\left(\rho \mathrm{NIR}+2.4^{*} \rho \mathrm{RED}+1\right)}$ & Jiang et al. (2008) \\
\hline GNDVI & $\frac{\rho \text { NIR- } \rho \text { GREEN }}{\rho N I R+\rho \text { GREEN }}$ & Gitelson et al. (1996) \\
\hline ISR & $\frac{\rho \mathrm{NIR}}{\rho \mathrm{SWIR}}$ & $\begin{array}{l}\text { Fernandes et al. } \\
\qquad(2003)\end{array}$ \\
\hline MTVI & $(1.2 *(1.2 * \rho N I R-\rho$ GREEN $)-2.5 * \rho R E D-\rho$ GREEN $)$ & $\begin{array}{l}\text { Haboudane et al. } \\
\qquad(2004)\end{array}$ \\
\hline $\mathrm{MTVI}_{2}$ & $\frac{1.5 *(1.2 *(\rho \text { NIR- } \rho \text { GREEN })-2.5 *(\rho \text { RED- } \rho \text { GREEN }))}{\sqrt{2 *(\rho \text { NIR }+1)^{2}-6 * \rho N I R+5 * \sqrt{\rho \text { RED-O.5 }}}}$ & $\begin{array}{l}\text { Haboudane et al. } \\
\qquad(2004)\end{array}$ \\
\hline $\mathrm{MVI}$ & $\frac{\rho \mathrm{NIR}-\rho \mathrm{SWIR}}{\rho \mathrm{NIR}+\rho \mathrm{SWIR}}$ & Gao (1996) \\
\hline NDVI & $\frac{\rho N I R-\rho \text { RED }}{\rho N I R+\rho \text { RED }}$ & Rouse et al. (1974) \\
\hline OSAVI & $\frac{\rho N I R-\rho \text { RED }}{(\rho N I R+\rho \text { RED }+1.6) * 1.16}$ & $\begin{array}{l}\text { Rondeaux et al. } \\
\qquad(1996)\end{array}$ \\
\hline SAVI & $\frac{(1+\mathrm{L})(\rho \mathrm{NIR}-\rho \mathrm{RED})}{\rho \mathrm{NIR}+\rho \mathrm{RED}+\mathrm{L}}$ & Huete (1988) \\
\hline SR & $\frac{\rho N I R}{\rho R E D}$ & Jordan (1969) \\
\hline WDRVI & $\frac{0.1 *(\rho \mathrm{NIR}-\rho \mathrm{RED})}{0.1 *(\rho \mathrm{NIR}-\rho \mathrm{RED})+(0.9 / 1.1)}$ & Gitelson (2004) \\
\hline \multicolumn{3}{|c|}{$\begin{array}{l}\text { Note: } \rho \text { BLUE: Blue band reflectance; } \rho \text { GREEN: Green band reflectance; } \rho \text { RED: Reflectance of red band; } \rho \text { NIR: Reflectance } \\
\text { of the near Infrared band; } \rho \text { SWIR: Reflectivity of the short-wave infrared band; L: constant that minimizes the effects of } \\
\text { the soil, used in this study the value of 0.50;ARVI: Atmospherically Resistant Vegetation Index; CLgreen: Green Chlorophyll } \\
\text { Index; DVI: Difference Vegetation Index; EVI: Enhanced Vegetation Index; EVI2: Enhanced Vegetation Index 2: GNDVI: } \\
\text { Green Normalized Difference Vegetation Index; ISR: Infrared Simple Ratio; MTVI: Modified Triangular Vegetation Index; } \\
\text { MTVI2: Modified Triangular Vegetation Index; MVI: Moisture Vegetation Index; NDVI: Normalized Difference Vegetation } \\
\text { Index; OSAVI: Optimized Soil Adjusted Vegetation Index; SAVI: Soil Adjusted Vegetation Index; SR: Simple Ratio } \\
\text { Vegetation Index; WDRVI: Wide Dynamic Range Vegetation Index. }\end{array}$} \\
\hline
\end{tabular}


The plots geo-referencing in the orbital images was carried out from the central point of the plots obtained with GPS. In a GIS (Geographic Information System) environment, a buffer with the same radius of the field-allocated plots was constructed. Then, using statistical tools it was possible to obtain the average value of each index per plot (Environmental Systems Research Institute, 2018).

\section{Regression models}

The correlation between LAI values and mean vegetation indices per plot was tested by the Pearson correlation analysis. The best-correlated indices were used in the construction of regression models by the Stepwise technique (Backward and Forward). The variables obtained in the images were considered as independent, while the LAI data were the dependent variables. The fitted models to the estimation of LAI by plot can be visualized in Table 2.

Table 2. Adjusted models for the estimation of LAl by plot $\left(\mathrm{m}^{2} \mathrm{~m}^{-2} 0.05 \mathrm{ha} \mathrm{a}^{-1}\right)$ using vegetation indices of the Landsat-8/OLI and Sentinel-2 / MSI sensors.

\begin{tabular}{|c|c|c|}
\hline Model & Equation & Reference \\
\hline \multicolumn{3}{|c|}{ Landsat-8 } \\
\hline 1 & $\begin{array}{c}\mathrm{I}=\beta_{0}+\beta_{1} * \mathrm{VI}+\beta_{2} * \mathrm{VI} 2+\beta_{3} * \mathrm{VI} 3+\beta_{4} * \mathrm{VI}^{2}+\beta_{5} * \mathrm{VI}^{4}+ \\
\beta_{6} * \mathrm{VI}^{5}+\beta_{7} * \mathrm{VI} 2^{2}+\beta_{8} * \mathrm{VI}_{2}{ }^{4}+\beta_{9} * \mathrm{VII}^{5}+\beta_{10} * \mathrm{VI}^{2}+ \\
\beta_{11} * \mathrm{VI}^{3}+\beta_{12} * \mathrm{VI}^{4}+\beta_{13} * \mathrm{VI}^{5}+\beta_{14} * \ln \mathrm{VI} 2^{2}+\beta_{15} * \exp \mathrm{VI}+ \\
\beta_{16} * \exp \mathrm{VI}^{2}+\beta_{17} * \exp \mathrm{VI}^{3}+\beta_{18} * \exp \mathrm{VI}^{2}+\beta_{19} * \exp \mathrm{VI}^{3}+ \\
\beta_{20} * \frac{1}{\mathrm{VI} 3}\end{array}$ & Stepwise 1 \\
\hline 2 & $\begin{array}{c}\mathrm{I}=\beta_{0}+\beta_{1} * \mathrm{VI}+\beta_{2} * \mathrm{VI} 2+\beta_{3} * \mathrm{VI} 2^{2}+\beta_{4} * \mathrm{VI}^{3}+\beta_{5} * \mathrm{VI}^{4}+ \\
\beta_{6} * \mathrm{VI}^{5}+\beta_{7} * \mathrm{VI}^{2}+\beta_{8} * \mathrm{VI}_{2}^{3}+\beta_{9} * \mathrm{VI}^{4}+\beta_{10} * \mathrm{VI}^{5}+ \\
\beta_{11} * \exp \mathrm{VI}+\beta_{12} * \exp \mathrm{VI}^{2}+\beta_{13} * \exp \mathrm{VI}^{3}+\beta_{14} * \frac{1}{\mathrm{VI}^{3}}+\beta_{15} * \frac{1}{\mathrm{VI}^{5}}+ \\
\beta_{16} * \frac{1}{\mathrm{VI}_{2}^{3}}+\beta_{17} * \frac{1}{\mathrm{VI}^{5}}+\beta_{18} * \mathrm{VI} * \mathrm{VI} 2+\beta_{19} * \frac{1}{\mathrm{VI}^{3}} * \frac{1}{\mathrm{VI} 2}\end{array}$ & Stepwise 2 \\
\hline 3 & $\begin{array}{c}\mathrm{I}=\beta_{0}+\beta_{1} * \mathrm{VI}+\beta_{2} * \mathrm{VI} 2+\beta_{3} * \mathrm{VI}^{2}+\beta_{4} * \mathrm{VI}^{3}+\beta_{5} * \mathrm{VI}^{4}+ \\
\beta_{6} * \mathrm{VI}^{5}+\beta_{7} * \mathrm{VI}^{2}+\beta_{8} * \mathrm{VI}^{3}+\beta_{9} * \mathrm{VI}^{4}+\beta_{10} * \mathrm{VI}^{5}+ \\
\beta_{11} * \exp \mathrm{VI}+\beta_{12} * \exp \mathrm{VI}^{2}+\beta_{13} * \exp \mathrm{VI}^{3}+\beta_{14} * \frac{1}{\mathrm{VI}^{3}}+\beta_{15} * \frac{1}{\mathrm{VI}^{5}}+ \\
\beta_{16} * \frac{1}{\mathrm{VI} 2^{4}}+\beta_{17} * \frac{1}{\mathrm{VI} 2^{5}}+\beta_{18} * \mathrm{VI} * \mathrm{VI} 2+\beta_{19} * \frac{1}{\mathrm{VI}^{4}} * \frac{1}{\mathrm{VI}}\end{array}$ & Stepwise 3 \\
\hline 4 & $\begin{aligned} \mathrm{I}= & \beta_{0}+\beta_{1} * \mathrm{VI}+\beta_{2} * \mathrm{VI}^{2}+\beta_{3} * \mathrm{VI}^{3}+\beta_{4} * \mathrm{VI}^{4}+ \\
& \beta_{5} * \mathrm{VI}^{5} \beta_{6} * \exp \mathrm{VI}^{2}+\beta_{7} * \frac{1}{\mathrm{VI}^{3}}+\beta_{8} * \frac{1}{\mathrm{VI}^{4}}\end{aligned}$ & Stepwise 4 \\
\hline 5 & $\begin{array}{c}\mathrm{I}=\beta_{0}+\beta_{1} * \mathrm{VI}+\beta_{2} * \mathrm{VI} 2+\beta_{3} * \mathrm{VI}^{2}+\beta_{4} * \mathrm{VI}^{4}+\beta_{5} * \mathrm{VI}^{5}+ \\
\beta_{6} * \mathrm{VI} 2^{2}+\beta_{7} * \mathrm{VI}^{4} \beta_{8} \mathrm{VI} 2^{5} \beta_{9} \ln \mathrm{VI}+\beta_{10} * \exp \mathrm{VI}+ \\
\beta_{11} * \exp \mathrm{VI}^{2}+\beta_{12} \exp \mathrm{VI}^{3}+\beta_{13} * \frac{1}{\mathrm{VI}^{2}}\end{array}$ & Stepwise 5 \\
\hline
\end{tabular}


Table 2. Continued..

3

4

5

\section{Sentinel-2}

Stepwise 1

$\mathrm{I}=\beta_{0}+\beta_{1} * \mathrm{VI}+\beta_{2} * \mathrm{VI} 2+\beta_{3} * \mathrm{VI}^{2}+\beta_{4} * \mathrm{VI}^{4}+\beta_{5} * \mathrm{VI}^{5}+$

$\beta_{6} * \mathrm{VI} 2^{2}+\beta_{7} * \mathrm{VI}^{4}+\beta_{8} * \mathrm{VI} 2^{5}+\beta_{9} * \mathrm{VII}^{2}+\beta_{10} * \mathrm{VI}^{3}+$

$\beta_{11} * \mathrm{VI}^{4}+\beta_{12} * \mathrm{VI} 3^{5}+\beta_{13} * \ln \mathrm{VI}+\beta_{14} * \ln \mathrm{VI} 2+\beta_{15} \sqrt{\mathrm{VI}^{5}}+$

$1 \quad \beta_{16} \sqrt{\mathrm{VI}^{3}}+\beta_{17} \sqrt{\mathrm{VI} 2^{5}}+\beta_{18} * \frac{1}{\mathrm{VI}}+\beta_{19} * \frac{1}{\mathrm{VI} 2}+\beta_{20} * \frac{1}{\mathrm{VI}^{3}}+\beta_{21} * \frac{1}{\mathrm{VI}^{5}}+$

$\beta_{22} * \frac{1}{\mathrm{VI}^{2}}+\beta_{23} * \frac{1}{\mathrm{VI}^{4}}+\beta_{24} * \frac{1}{\mathrm{VI}^{2}}+\beta_{25} * \frac{1}{\mathrm{VI}^{3}}+\beta_{26^{2}} * \frac{1}{\mathrm{VI}^{5}}+$

$\beta_{27} * \mathrm{VI} * \mathrm{VI} 2+\beta_{28} * \mathrm{VI} * \mathrm{VI} 3$

$\mathrm{I}=\beta_{0}+\beta_{1} * \mathrm{VI}+\beta_{2} * \mathrm{VI} 2+\beta_{3} * \mathrm{VI} 3+\beta_{4} * \mathrm{VI}^{2}+\beta_{5} * \mathrm{VI}^{3}+$

$\beta_{6} * \mathrm{VI}^{4}+\beta_{7} * \mathrm{VI}^{5}+\beta_{8} * \mathrm{VI}^{2}+\beta_{9} * \mathrm{VI}^{3}+\beta_{10} * \mathrm{VI}^{4}+$

$\beta_{11} * \mathrm{VI}^{5}+\beta_{12} * \mathrm{VI}^{2}+\beta_{13} * \mathrm{VI}^{3}+\beta_{14} * \mathrm{VI}^{4}+\beta_{15} * \mathrm{VI}^{5}+$

$\beta_{16} * \ln \mathrm{VI}+\beta_{17} * \ln \mathrm{VI} 2+\beta_{18} * \ln \mathrm{VI} 3+\beta_{19} * \sqrt{\mathrm{VI}^{3}}+\beta_{20} * \sqrt{\mathrm{VI}^{5}}+$

$\beta_{21} * \sqrt{\mathrm{VI} 2^{3}}+\beta_{22} * \sqrt{\mathrm{VI}^{5}}+\beta_{23} * \sqrt{\mathrm{VI}^{5}}+\beta_{24} * \frac{1}{\mathrm{VI}}+\beta_{25} * \frac{1}{\mathrm{VI} 2}+$

$\beta_{26} * \frac{1}{\mathrm{VI} 3}+\beta_{27} * \frac{1}{\mathrm{VI}^{2}}+\beta_{28} * \frac{1}{\mathrm{VI}^{3}}+\beta_{29} * \frac{1}{\mathrm{VI}^{4}}+\beta_{30} * \frac{1}{\mathrm{VI}^{5}}+\beta_{31} * \frac{1}{\mathrm{VI}^{2}}+$

Stepwise 2

$\beta_{32} * \frac{1}{\mathrm{VI}^{4}}+\beta_{33} * \frac{1}{\mathrm{VI}^{2}}+\beta_{34} * \frac{1}{\mathrm{VI}^{3}}+\beta_{35} * \frac{1}{\mathrm{VI}^{5}}+\beta_{36} * \mathrm{VI} * \mathrm{VI} 2+$

$$
\beta_{37} * \mathrm{VI} * \mathrm{VI} 3
$$

$\mathrm{I}=\beta_{0}+\beta_{1} * \mathrm{VI}+\beta_{2} * \mathrm{VI} 2+\beta_{3} * \mathrm{VI}^{2}+\beta_{4} * \mathrm{VI}^{3}+\beta_{5} * \mathrm{VI}^{4}+$ $\beta_{6} * \mathrm{VI}^{5}+\beta_{7} * \mathrm{VI} 2^{2}+\beta_{8} * \mathrm{VI} 2^{3}+\beta_{9} * \mathrm{VI}^{4}+\beta_{10} * \mathrm{VI} 2^{5}+$

$\beta_{11} * \ln \mathrm{VI}+\beta_{12} * \ln \mathrm{VI} 2+\beta_{13} * \sqrt{\mathrm{VI}^{3}}+\beta_{14} * \sqrt{\mathrm{VI}^{5}}+\beta_{15} * \frac{1}{\mathrm{VI}}+$

$$
\begin{gathered}
\beta_{16} * \frac{1}{\mathrm{VI} 2}+\beta_{17} * \frac{1}{\mathrm{VI} 3}+\beta_{18} * \frac{1}{\mathrm{VI}^{2}}+\beta_{19} * \frac{1}{\mathrm{VI}^{3}}+\beta_{20} * \frac{1}{\mathrm{VI}^{4}}+ \\
\beta_{21} * \frac{1}{\mathrm{VI}^{5}}+\beta_{22} * \frac{1}{\mathrm{VI}^{2}}+\beta_{23} * \frac{1}{\mathrm{VI}^{4}}+\beta_{24} * \mathrm{VI} * \mathrm{VI} 2
\end{gathered}
$$

$\mathrm{I}=\beta_{0}+\beta_{1} * \mathrm{VI}+\beta_{2} * \mathrm{VI} 2+\beta_{3} * \mathrm{VI}^{2}+\beta_{4} * \mathrm{VI}^{3}+\beta_{5} * \mathrm{VI}^{4}+$

$\beta_{6} * \mathrm{VI}^{5}+\beta_{7} * \mathrm{VI}^{2}+\beta_{8} * \mathrm{VI}^{3}+\beta_{9} * \mathrm{VI}^{4}+\beta_{10} * \mathrm{VI}^{5}+$

$\beta_{11} * \ln \mathrm{VI}+\beta_{12} * \ln \mathrm{VI} 2+\beta_{13} * \sqrt{\mathrm{VI}^{3}}+\beta_{14} * \sqrt{\mathrm{VI}^{5}}+\beta_{15} * \sqrt{\mathrm{VI}^{3}}+$

$$
\beta_{16} * \sqrt{\mathrm{VI}^{5}}+\beta_{17} * \frac{1}{\mathrm{VI}}+\beta_{18} * \frac{1}{\mathrm{VI}^{2}}+\beta_{19} * \frac{1}{\mathrm{VI}^{3}}+\beta_{20} * \frac{1}{\mathrm{VI}^{4}}+
$$

Stepwise 4

$$
\beta_{21} * \frac{1}{\mathrm{VI}^{5}}+\beta_{22} * \frac{1}{\mathrm{VI} 2^{2}}+\beta_{23} * \frac{1}{\mathrm{VI}^{3}}+\beta_{24} * \frac{1}{\mathrm{VI} 2^{4}}+\beta_{25} * \frac{1}{\mathrm{VI}^{5}}+
$$

$$
\beta_{26} * \mathrm{VI} * \mathrm{VI} 2+\beta_{27} * \ln \mathrm{VI} * \ln \mathrm{VI} 2+\beta_{28} * \frac{1}{\mathrm{VI}^{3}} * \frac{\mathrm{I}}{\mathrm{VI} 2}
$$

$\mathrm{I}=\beta_{0}+\beta_{1} * \mathrm{VI}+\beta_{2} * \mathrm{VI}^{4}+\beta_{3} * \mathrm{VI}^{5}+\beta_{4} * \mathrm{VI} 2^{2}+\beta_{5} * \mathrm{VI} 2^{5}+$

$$
\beta_{6} * \sqrt{\mathrm{VI}^{3}}+\beta_{7} * \sqrt{\mathrm{VI}^{5}}+\beta_{8} * \frac{1}{\mathrm{VI}}+\beta_{9} * \frac{1}{\mathrm{VI}^{2}}+\beta_{10} * \frac{1}{\mathrm{VI}^{3}}+
$$

$$
\begin{gathered}
\beta_{11} * \frac{1}{\mathrm{VI}^{5}}+\beta_{12} * \frac{1}{\mathrm{VI}^{2}}+\beta_{13} * \frac{1}{\mathrm{VI}^{3}}+\beta_{14} * \frac{1}{\mathrm{VI}^{4}}+ \\
\beta_{15} * \frac{1}{\mathrm{VI}^{5}}+\beta_{16} * \mathrm{VI} * \mathrm{VI} 2
\end{gathered}
$$

Stepwise 3

Stepwise 5

Note: I: LAI by plot ( $\mathrm{m}^{2} \mathrm{~m}^{-2}$ 0.05ha); âi: parameters to be estimated; VI: Vegetation index; VI2: Vegetation index 2; VI3: Vegetation index 3; In: natural logarithm based on the constant e (2.71828182845904); EXP: natural exponential function. 
The criteria for choosing the best model were the following: higher adjusted coefficient of determination ( $R^{2}$ adjusted) (Equation 1 ), lower standard error values of the estimate (Syx\%) (Equation 2 and 3), Akaike Information Criterion (AIC) Equation 4), Bayesian Information Criterion (BIC) (Equation 5) and Root Mean Squared Error (RMSE) (Equation 6).

$$
\begin{aligned}
& \mathrm{R}^{2} \text { aj }=1-\left\{\left(1-\mathrm{R}^{2}\right) *\left(\frac{\mathrm{n}-1}{\mathrm{n}-\mathrm{p}}\right)\right\} \\
& \mathrm{Syx}=\sqrt{\frac{\sum(\mathrm{y}-\mathrm{yi})^{2}}{\mathrm{n}-\mathrm{p}}} \\
& \mathrm{Syx}=\frac{\mathrm{Syx}}{\widehat{\mathrm{Y}}} * 100 \\
& \mathrm{AIC}=\mathrm{n} * \ln (\mathrm{SQ} \text { res })-\mathrm{n} * \ln (\mathrm{n})+2 \mathrm{p} \\
& \mathrm{BIC}=-2 \log \left(\mathrm{L}_{\mathrm{p}}\right)+[(\mathrm{p}+1)+1] \log (\mathrm{n}) \\
& \mathrm{RMSE}=\sqrt{\frac{\sum(\mathrm{y}-\mathrm{yi})^{2}}{\mathrm{n}}}
\end{aligned}
$$

Note: $\mathrm{R}^{2}$ aj: adjusted coefficient of determination; $\mathrm{n}$ : number of observations; $\mathrm{p}$ : number of parameters of the equation; Syx: standard error of estimate $\left(\mathrm{m}^{2} \mathrm{~m}^{-2} 0.05 \mathrm{ha} \mathrm{a}^{-1}\right)$; y: LAl observed $\left(\mathrm{m}^{2} \mathrm{~m}^{-2} 0.05 \mathrm{ha}^{-1}\right)$; yi: LAl estimated $\left(\mathrm{m}^{2} \mathrm{~m}^{-2} 0.05 \mathrm{ha}^{-1}\right)$; Syx (\%):standard error of the percentage estimate (\%); $\hat{y}$ : mean of the observed values $\left(\mathrm{m}^{2} \mathrm{~m}^{-2} 0.05 \mathrm{ha}^{-1}\right) ; \mathrm{p}$ : number of model parameters; SQres: Sum of Squares of the residues obtained by ANOVA; Lp: maximum likelihood function of the model; RMSE: Root Mean Square Error $\left(\mathrm{m}^{2} \mathrm{~m}^{-2} 0.05 \mathrm{ha}^{-1}\right)$.

\section{Statistical analyses}

Normality was assessed using the Shapiro-Wilk test. The design was completely randomized with three treatments (LAI predicted by field measurements, LAl estimated by vegetation indices from Landsat-8/OLI and LAl estimated by the vegetation indices of Sentinel-2/MSI). The analyses were performed in software $\mathrm{R}$ version 3.4.1 (R Core Team, 2018).

\section{RESULTS AND DISCUSSION}

The LAl values per plot calculated varied from 2.51 to $5.32\left(\mathrm{~m}^{2} \mathrm{~m}^{-2} 0.05 \mathrm{ha}^{-1}\right)$, with a mean of $3.65\left(\mathrm{~m}^{2} \mathrm{~m}^{-2} 0.05 \mathrm{ha}^{-1}\right)$ as shown in Figure 2:

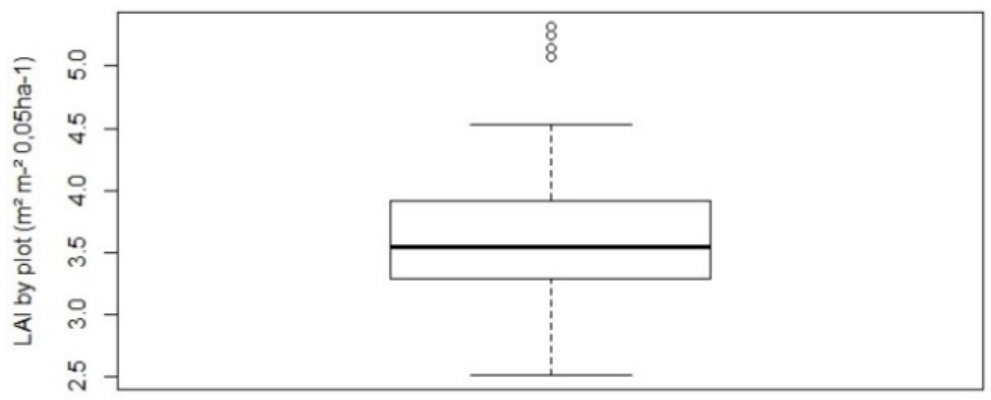

Figure 2. Descriptive statistics for the LAI variable per plot of a Pinus taeda L. and Cupressus lusitanica Mill. forest in Campo Belo do Sul - SC. 
The correlation among vegetation indices with LAI per plot (Table 3) revealed that the highest correlation was observed in Sentinel-2 indices with 0.4368 for MTVI2. For the Landsat-8 data, the MTVI index was better correlated with the LAI, with a value of 0.4097.

Table 3. Pearson correlation coefficients between LAI per plot $\left(\mathrm{m}^{2} \mathrm{~m}^{-2} 0.05\right.$ ha- $\left.{ }^{-1}\right)$ and vegetation indices derived from Landsat-8 and Sentinel-2 images for a forest of Pinus taeda L. and Cupressus lusitanica Mill. located in Campo Belo do Sul - SC.

\begin{tabular}{ccc}
\hline & & LAI \\
\hline VI & Landsat-8 & Sentinel-2 \\
LAI & 1 & 1 \\
ARVI & 0.3555 & 0.4090 \\
CHG & 0.2743 & 0.3441 \\
DVI & $0.3826^{*}$ & 0.4123 \\
EVI & -0.3518 & $0.4144^{*}$ \\
EVI2 & 0.2499 & 0.4135 \\
GNDVI & 0.2758 & 0.3528 \\
ISR & -0.1099 & -0.0490 \\
MTVI & $0.4097^{*}$ & $0.4302^{*}$ \\
MTVI2 & 0.0071 & $0.4368^{*}$ \\
MVI & -0.1106 & -0.0840 \\
NDVI & 0.1854 & 0.3590 \\
OSAVI & 0.3121 & 0.4128 \\
SAVI & 0.2437 & 0.4138 \\
SR & 0.1791 & 0.3701 \\
WDRVI & $0.3808^{*}$ & 0.4124
\end{tabular}

Where: VI: Vegetation index; LAI: LAI by plot $\left(\mathrm{m}^{2} \mathrm{~m}^{-2} 0.05 \mathrm{ha}\right)$. *Significant correlation at $5 \%$ probability.

The correlation among vegetation indexes and LAI per plot revealed that the indices using spectral bands corresponding to red and infrared were more sensitive to LAl. This fact is associated with higher reflectance in this spectral range, caused by a higher number of leaves and higher LAl.

As obtained by Almeida et al. (2015), the higher the vegetation indexes, the higher the LAI per plot. Except for ISR, MVI, and EVI indexes (only for Landsat-8), there was a positive correlation between biophysical parameter and spectral data.

In other studies, other indices were well correlated with LAI, such as SAVI in Biudes et al. (2014), WDRVI in Nguy-Robertson et al. (2014) and NDVI in Almeida et al. (2015). The MTVI2 index, the best correlated index for Sentinel-2/MSI, was also higher than other indexes in Kross et al. (2015). The feature of presenting sensitivity to the angle of leaf slope is one of the factors that may have contributed to this correlation, according to Liu et al. (2012). On the other hand, the SR index generated reliable estimates of LAI and canopy chlorophyll density in areas with low density vegetation, such as in Broge \& Leblanc (2001) and high-density vegetation, such as NguyRobertson et al. (2012).

The regression models fitting for the estimation of LAI by plot (Table 4 and Figure 3) from the best correlated vegetation indexes demonstrate moderate fitting metrics. 
Table 4. Statistics of the adjusted regression models with the indices correlated with the Foliar Area Index for a Pinus taeda L. and Cupressus lusitanica Mill. forest located in Campo Belo do Sul - SC.

\begin{tabular}{cccccccc}
\hline \multicolumn{7}{c}{ Landsat-8 } \\
\hline Model & $\mathbf{R}^{2}$ aj & Syx & Syx (\%) & $\mathbf{F}$ & AIC & BIC & RMSE \\
\hline 1 & 0.6701 & 0.3424 & 9.30 & 4.772 & 41.83 & 80.66 & 0.228 \\
2 & 0.6981 & 0.3275 & 8.90 & 5.747 & 38.48 & 73.95 & 0.231 \\
3 & 0.6711 & 0.3512 & 9.54 & 6.234 & 43.22 & 82.58 & 0.206 \\
4 & 0.0955 & 0.5669 & 15.21 & 1.316 & 80.88 & 106.21 & 0.414 \\
5 & 0.7321 & 0.3085 & 8.38 & 8.106 & 32.99 & 61.71 & 0.241 \\
\hline & & & & Sentinel-2 & & & \\
\hline Model & $\mathbf{R}^{2}$ aj & Syx & Syx (\%) & $\mathbf{F}$ & AIC & BIC & RMSE \\
\hline 1 & 0.9134 & 0.1754 & 4.75 & 14.272 & -24.11 & 31.63 & 0.079 \\
2 & 0.6624 & 0.3463 & 9.41 & 3.068 & -13.15 & 52.72 & 0.077 \\
3 & 0.7466 & 0.3012 & 8.15 & 5.105 & 25.56 & 76.23 & 0.156 \\
4 & 0.6654 & 0.3448 & 9.37 & 3.502 & 29.95 & 85.68 & 0.152 \\
5 & 0.3739 & 0.4716 & 12.81 & 2.109 & 67.45 & 106.29 & 0.343 \\
\hline
\end{tabular}

Note: $\mathrm{R}^{2}$ adj: adjusted coefficient of determination; Syx: standard error of estimate ( $\left.\mathrm{m}^{2} \mathrm{~m}^{-2} 0.05 \mathrm{ha}\right)$; Syx (\%): standard error of the percentage estimate; F: $\mathrm{F}$ test at 5\% probability; AIC: Akaike Information Criterion; BIC: Bayesian Information Criterion; RSME: Root Mean Square Error $\left(\mathrm{m}^{2} \mathrm{~m}^{-2} 0.05 \mathrm{ha}^{-1}\right)$.

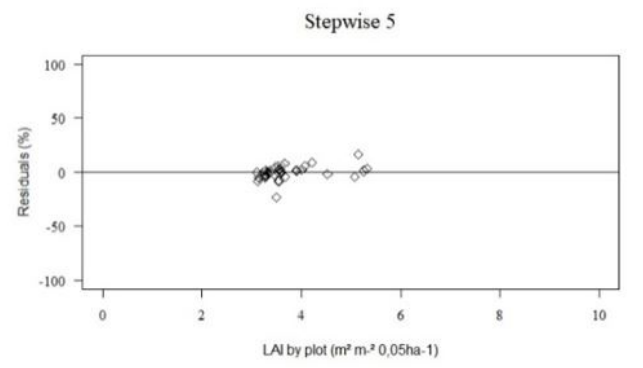

A)

Figure 3. Graphical dispersion of the residuals of the adjusted models for the estimation of Foliar Area Index through vegetation indices from Landsat-8/OLI (A) and Sentinel-2/MSI (B).

When analyzing Table 4, it is possible to perceive that the metrics of the models fitted with the Landsat-8/OLI data were lower than those from Sentinel-2/MSI. This fact can be verified by the higher adjusted $\mathrm{R}^{2}$ and smaller statistics of Syx, AIC, BIC, and RMSE. The residuals graphical distribution (Figure 2) reinforces this idea, since the model developed using Sentinel-2/MSI data had its residuals distributed around the regression line, while the Landsat-8/OLI model presented discrepant data (under and super estimates).

In contrast to this scenario, the statistical analysis revealed that there was no difference between the LAl predicted by the field measurements, with the Landsat-8/OLI and Sentinel-2/MSI derived indices.

The fitted model's accuracy was influenced by the structural pattern and stratification of the forest canopy, causing differences in the indices and the absorption of the electromagnetic radiation (Bréda, 2003). Another factor that interfered with the results involves the spatial resolution of the sensors used. Wang And Liang (2008) suggest that sensors with higher spatial resolution can provide more details about land surface objects, favoring better estimates of LAI.

The development of standard functions of sensitivity and performance evaluation for the relationship between vegetation index and LAI in different soil types was performed by Gonsamo (2011). Using data from the PROSAIL (PROSPECT and SAIL radiative transfer models) 
model, the authors concluded that the Infrared Simple Ratio (ISR) and Reduced Infrared Simple Ratio (RISR) scores had the best fitting performance in the association with LAI.

The definition of bands number and spectral regions of the Sentinel-2/MSI sensor for the estimation of LAl in cultures was investigated by Richter et al. (2012). For that, field data were explored using models of radioactive inversion and neural networks. The results showed that LAl can be estimated using two or three infrared and red-edge spectral bands or vegetation indices. The authors also highlighted the potential of the Sentinel-2/MSI sensor for agricultural and forestry applications.

The LAl estimates obtained from field campaigns were compared with estimates derived from MODIS (MODerate Resolution Imaging Spectroradiometer) and Landsat-5/TM sensors in tropical forests and different seasonal scales by Biudes et al. (2014). The remotely located data were efficient in the LAl estimates; however, they should be used and interpreted with caution. This can be explained by the limitations and complexities associated with these data.

The fitting of regression models among leaf area values measured in field of Eucalyptys grandis $\mathrm{x}$ urophylla forests and vegetation indices derived from Landsat-5/TM images were evaluated by Almeida et al. (2015). Using a simple linear relationship with NDVI and multiple linear relation, the fitted models had $R^{2}$ of 0.60 to 0.75 , which are good results. The NDVI presented saturation, but it generated the best models to estimate the LAI of the stand assessed by these authors. With the SAVI data, the models had high $\mathrm{R}^{2}$ and larger mean squared errors.

Härkönen et al. (2015) examined the feasibility of producing a high-resolution LAI map based on inventory data, allometric equations, and Landsat-5/TM images. This methodology revealed that it can be used for the production of LAI maps in large regions with ecological applications.

Combinations of bands and vegetation indices from Sentinel-2/MSI images for the estimation of canopy biophysical properties (LAl and biomass) of boreal forests was explored by Majasalmi \& Rautiainen (2016). The data demonstrated that the red and infrared bands and the IRECI (Inverted Red-edge Chlorophyll Index) and S2REP (Sentinel-2 red-edge Position) indices resulted in the best LAl estimates.

The research conducted by Korhonen et al. (2017) was the first one to compare estimates of biophysical variables such as LAI from Landsat-8/OLI and Sentinel-2/MSI data for boreal forests to vegetation indices. Models developed with the red-edge (Sentinel-2/MSI) bands resulted in lower RMSE than the Landat-8/OLI models. The authors highlighted the potential of using Sentinel-2 in the estimation of biophysical parameters of forests.

The spatial variation of the LAl over a forest cover area of a mountainous landscape in central Japan from Landsat-8/OLI data was performed by Melnikova et al. (2018). The fitted simple model had significant estimates and it was considered applicable to the assessed forest ecosystems. However, the authors recommend validating and improving this model using larger samples with defined sampling strategies.

\section{CONCLUSION}

The model that most accurately and precisely estimated the Leaf Area Index per plot of Pinus taeda L. and Cupressus lusitanica Mill. was a result of the vegetation indexes obtained from the Sentinel-2/MSI sensor, with an adjusted $\mathrm{R}^{2}$ of 0.9134 and an error of $4.75 \%$.

There was no significant difference between the LAI obtained by the field campaign and the LAI estimated by the spectral data (Landsat-8/OLI and Sentinel-2/MSI). However, it is recommended to test this methodology using sensors of high spatial resolution and with other species of the genus Pinus spp. and Cupressus spp.

\section{REFERENCES}

Almeida, A. Q. D., Ribeiro, A., Delgado, R. C., Rody, Y. P. R., Oliveira, A. S. D., \& Leite, F. P. (2015). Índice de área foliar de Eucalyptus estimado por índices de vegetação utilizando imagens TM - Landsat 5. Floresta e Ambiente, 22(3), 368-376. http://dx.doi.org/10.1590/2179-8087.103414. 
Alvares, C. A., Stape, J. L., Sentelhas, P. C., Gonçalves, J. L. M., \& Sparovek, G. (2013). Köppen's climate classification map for Brazil. Meteorologische Zeitschrift, 22(6), 711-728. http://dx.doi.org/10.1127/0941-2948/2013/0507.

Baret, F., \& Buis, S. (2008). Estimating canopy characteristics from remote sensing observations: review of methods and associated problems. In S. Liang (Ed.), Advances in land remote sensing (pp. 173-201). New York: Springer. http://dx.doi.org/10.1007/978-1-4020-6450-0_7.

Biudes, M. S., Machado, N. G., Danelichen, V. H. M., Souza, M. C., Vourlitis, G. L., \& Nogueira, J. S. (2014). Ground and remote sensing-based measurements of leaf area index in a transitional forest and seasonal flooded forest in Brazil. International Journal of Biometeorology, 58(6), 1181-1193. PMid:23943204. http://dx.doi.org/10.1007/s00484-013-0713-4.

Bolstad, P. V., Vose, J. M., \& Mcnulty, S. G. (2001). Forest productivity, leaf area, and terrain in southern Appalachian deciduous forests. Forest Science, 47, 419-427.

Bréda, N. J. J. (2003). Ground-based measurements of leaf area index: a review of methods, instruments and current controversies. Journal of Experimental Botany, 54(392), 2403-2417. PMid:14565947. http://dx.doi.org/10.1093/jxb/erg263.

Broge, N. H., \& Leblanc, E. (2001). Comparing prediction power and stability of broadband and hyper spectral vegetation indices for estimation of green leaf area index and canopy chlorophyll density. Remote Sensing of Environment, 76(2), 156-172. http://dx.doi.org/10.1016/S0034-4257(00)00197-8.

Cho, M. A., Skidmore, A., Corsi, F., Van Wieren, S. E., \& Sobhan, I. (2007). Estimation of green grass/herb biomass from airborne hyperspectral imagery using spectral indices and partial least squares regression. International Journal of Applied Earth Observation and Geoinformation, 9(4), 414-424. http://dx.doi.org/10.1016/j.jag.2007.02.001.

Clevers, J., Kooistra, L., \& Van den Brande, M. (2017). Using sentinel-2 data for retrieving lai and leaf and canopy chlorophyll content of a potato crop. Remote Sensing, 9(405), 1-15. http://dx.doi.org/10.3390/rs9050405.

Dorigo, W. A., Zurita-Milla, R., De Wit, A. J. W., Brazile, J., Singh, R., \& Schaepman, M. E. (2007). A review on reflective remote sensing and data assimilation techniques for enhanced agroecosystem modeling. International Journal of Applied Earth Observation and Geoinformation, 9(2), 165-193. http://dx.doi.org/10.1016/j.jag.2006.05.003.

Environmental Systems Research Institute - ESRI. (2018). ArcGIS Professional GIS for the desktop, version 10.4.1. Redlands: ESRI. Retrieved in 2018, May 20, from https://support.esri.com/en/Products/Desktop/arcgisdesktop/arcmap/10-4-1

European Space Agency - ESA. (2010). GMES Sentinel-2 mission required document. Paris: ESA. Retrieved in 2018, March 22, from http://esamultimedia.esa.int/docs/GMES/Sentinel-2/MRD.pdf

Fernandes, R., Butson, C., Leblanc, S., \& Latifovic, R. (2003). Landsat-5 TM and derived from SPOT-4 Vegetation data. Canadian Journal of Remote Sensing, 29, 241-258. http://dx.doi.org/10.5589/m02-092.

Gao, B. C. (1996). NDWI a normalized difference water index for remote sensing of vegetation liquid water from space. Remote Sensing of Environment, 58(3), 257-266. http://dx.doi.org/10.1016/S0034-4257(96)00067-3.

Gitelson, A. A. (2004). Wide dynamic range vegetation index for remote quantification of biophysical characteristics of vegetation. Journal of Plant Physiology, 161(2), 165-173. PMid:15022830. http://dx.doi.org/10.1078/0176-1617-01176.

Gitelson, A. A., Verma, S. B., Vi, A. A., Rundquist, D. C., Keydan, G., Leavitt, B., Arkebauer, T. J., Burba, G. G., \& Suyker, A. E. (2003a). Novel technique for remote estimation of $\mathrm{CO}_{2}$ flux in maize. Geophysical Research Letters, 30(9), 1486-1489. http://dx.doi.org/10.1029/2002GL016543.

Gitelson, A. A., Viña, A., Arkebauer, T. J., Rundquist, D. C., Keydan, G., \& Leavitt, B. (2003b). Remote estimation of leaf area index and green leaf biomass in maize canopies. Geophysical Research Letters, 30(5), 1248-1251. http://dx.doi.org/10.1029/2002GL016450.

Gitelson, A. A., Kaufman, Y. J., \& Merzlyak, M. N. (1996). Use of a green channel in remote sensing of global vegetation from EOS-MODIS. Remote Sensing of Environment, 58(3), 289-298. http://dx.doi.org/10.1016/S0034-4257(96)00072-7.

Gonsamo, A. (2011). Normalized sensitivity measures for leaf area index estimation using three-band spectral vegetation indices. International Journal of Remote Sensing, 32(7), 2069-2080. http://dx.doi.org/10.1080/01431161.2010.502153.

Haboudane, D., Miller, J. R., Pattey, E., Zarco-Tejada, P. J., \& Strachan, I. B. (2004). Hyperspectral vegetation indices and novel algorithms for predicting green LAI of crop canopies: modeling and validation in the context of precision agriculture. Remote Sensing of Environment, 90(3), 337-352. http://dx.doi.org/10.1016/j.rse.2003.12.013. 
Härkönen, S., Lehtonen, A., Manninen, T., Tuominen, S., \& Peltoniemi, M. (2015). Estimating forest leaf area index using satellite images: comparison of k-NN based Landsat-NFI LAI with MODIS-RSR based LAI product for Finland. Boreal Environment Research, 20, 181-195.

Heiskanen, J. (2011). Estimating aboveground tree biomass and leaf area index in a mountain birch forest using ASTER satellite data. International Journal of Remote Sensing, 27(6), 1135-1158. http://dx.doi.org/10.1080/01431160500353858.

Huete, A. R. (1988). A soil-adjusted vegetation index (SAVI). Remote Sensing of Environment, 25(3), 295-309. http://dx.doi.org/10.1016/0034-4257(88)90106-X.

Huete, A. R., Liu, H. Q., \& Leeuwen, W. V. (1997). A comparison of vegetation indices over a global set of TM Images for EOS-MODIS. Remote Sensing of Environment, 59(3), 440-451. http://dx.doi.org/10.1016/S00344257(96)00112-5.

Indústria Brasileira de Árvores - IBÁ. (2017). Relatório 2017. São Paulo: IBÁ. Retrieved in 2018, July 14, from http://iba.org/images/shared/Biblioteca/IBA_RelatorioAnual2017.pdf

Jiang, Z., Huete, A. R., Didan, K., \& Miura, T. (2008). Development of a two-band enhanced vegetation index without a blue band. Remote Sensing of Environment, 112(10), 3833-3845. http://dx.doi.org/10.1016/j.rse.2008.06.006.

Jordan, C. F. (1969). Derivation of leaf-area index from quality of light on the forest floor. Ecology, 50(4), 663-666. http://dx.doi.org/10.2307/1936256.

Kaufman, Y. J., \& Tanré, D. (1992). Atmospherically resistant vegetation index (ARVI) for EOS-MODIS. IEEE Transactions on Geoscience and Remote Sensing, 30(2), 261-270. http://dx.doi.org/10.1109/36.134076.

Korhonen, L., Hadi, Packalen, P., \& Rautiainen, M. (2017). Comparison of Sentinel-2 and Landsat 8 in the estimation of boreal forest canopy cover and leaf area index. Remote Sensing of Environment, 195, 259-274. http://dx.doi.org/10.1016/j.rse.2017.03.021.

Kross, A., McNairn, H., Lapen, D., Sunohara, M., \& Champagne, C. (2015). Assessment of RapidEye vegetation indices for estimation of leaf area index and biomass in corn and soybean crops. International Journal of Applied Earth Observation and Geoinformation, 34, 235-248. http://dx.doi.org/10.1016/j.jag.2014.08.002.

Landsberg, J. J., \& Sands, P. (2011). Physiological ecology of forest production: principles, processes and models (Terrestrial Ecology Series, No. 4). New York: Academic Press.

Liu, Y., Liu, R., \& Chen, J. M. (2012). Retrospective retrieval of long-term consistent global leaf area index (19812011) from combined AVHRR and MODIS data. Journal of Geophysical Research, 117(G4). http://dx.doi.org/10.1029/2012JG002084.

Majasalmi, T., \& Rautiainen, M. (2016). The potential of Sentinel-2 data for estimating biophysical variables in a boreal forest: a simulation study. Remote Sensing Letters, 7(5), 427-436. http://dx.doi.org/10.1080/2150704X.2016.1149251.

Majasalmi, T., Rautiainen, M., Stenberg, P., \& Lukeš, P. (2013). An assessment of ground reference methods for estimating LAI of boreal forests. Forest Ecology and Management, 292, 10-18. http://dx.doi.org/10.1016/j.foreco.2012.12.017.

Melnikova, I., Awaya, Y., Saitoh, T., Muraoka, H., \& Sasai, T. (2018). Estimation of leaf area index in a mountain forest of central Japan with a 30-m spatial resolution based on landsat operational land imager imagery: an application of a simple model for seasonal monitoring. Remote Sensing, 10(179), 1-24. http://dx.doi.org/10.3390/rs10020179.

Myneni, R. B., Ramakrishna, R., Nemani, R., \& Running, S. W. (1997). Estimation of global leaf area index and absorbed PAR using radiative transfer models. IEEE Transactions on Geoscience and Remote Sensing, 35(6), 1380-1393. http://dx.doi.org/10.1109/36.649788.

Nagai, S., Inoue, T., Ohtsuka, T., Kobayashi, H., Kurumado, K., Muraoka, H., \& Nasahara, K. N. (2014). Relationship between spatio-temporal characteristics of leaf-fall phenology and seasonal variations in near surface-and satellite-observed vegetation indices in a cool-temperate deciduous broad-leaved forest in Japan. International Journal of Remote Sensing, 35(10), 3520-3536. http://dx.doi.org/10.1080/01431161.2014.907937.

Nasahara, K. N., Muraoka, H., Nagai, S., \& Mikami, H. (2008). Vertical integration of leaf area index in a Japanese deciduous broad-leaved forest. Agricultural and Forest Meteorology, 148(6-7), 1136-1146. http://dx.doi.org/10.1016/j.agrformet.2008.02.011.

Nguy-Robertson, A. L., Gitelson, A. A., Peng, Y., Viña, A., Arkebauer, T. J., \& Rundquist, D. C. (2012). Green Leaf Area Index estimation in maize and soybean: combining vegetation indices to achieve maximal sensitivity. Biometry, Modeling \& Statistics, 104(5), 1336-1347. http://dx.doi.org/10.2134/agronj2012.0065.

Nguy-Robertson, A. L., Peng, Y., Gitelson, A. A., Arkebauer, T. J., Pimstein, A., Herrmann, I., Karnieli, A., Rundquist, D. C., \& Bonfil, D. J. (2014). Estimating green LAl in four crops: potential of determining optimal spectral 
bands for a universal algorithm. Agricultural and Forest Meteorology, 192-193, 140-148.

http://dx.doi.org/10.1016/j.agrformet.2014.03.004

R Core Team. (2018). R: a language and environment for statistical computing. Vienna: R Foundation for Statistical Computing. Retrieved in 2018, April 16, from https://www.R-project.org

Richardson, A. J., \& Wegand, C. L. (1977). Distinguishing vegetation from soil background information. Photogrammetric Engineering and Remote Sensing, 43(12), 1541-1552.

Richter, K., Hank, T. B., Vuolo, F., Mauser, W., \& D'Urso, D. (2012). Optimal exploitation of the Sentinel-2 spectral capabilities for crop leaf area index mapping. Remote Sensing, 4(3), 561-582. http://dx.doi.org/10.3390/rs4030561.

Rody, H. P., Ribeiro, A., Pezzopane, J. E. M., Glerianijm, J. M., Almeida, A. Q., \& Leite, F. P. (2014). Estimates of the leaf area index (LAl) using LAl-2000 and hemispherical photos in Eucalyptus plantations. Ciência Florestal, 4, 923-932.

Rondeaux, G., Steven, M., \& Baret, F. (1996). Optimization of soil-adjusted vegetation indices. Remote Sensing of Environment, 55(2), 95-107. http://dx.doi.org/10.1016/0034-4257(95)00186-7.

Rouse, J. W., Haas, R. H., Deering, D. W., \& Schell, J. A. (1974). Monitoring the vernal advancement and retrogradation (green wave effect) of natural vegetation (Progress Report RSC, No. 1978-2). College Station: Texas A\&M University.

Roy, D. P., Wulder, M. A., Loveland, T. R., C.e, W., Allen, R. G., Anderson, M. C., Helder, D., Irons, J. R., Johnson, D. M., Kennedy, R., Scambos, T. A., Schaaf, C. B., Schott, J. R., Sheng, Y., Vermote, E. F., Belward, A. S., Bindschadler, R., Cohen, W. B., Gao, F., Hipple, J. D., Hostert, P., Huntington, J., Justice, C. O., Kilic, A., Kovalskyy, V., Lee, Z. P., Lymburner, L., Masek, J. G., McCorkel, J., Shuai, Y., Trezza, R., Vogelmann, J., Wynne, R. H., \& Zhu, Z. (2014). Landsat-8: science and product vision for terrestrial global chang research. Remote Sensing of Environment, 145, 154-172. http://dx.doi.org/10.1016/j.rse.2014.02.001.

Sasai, T., Saigusa, N., Nasahara, K. N., Ito, A., Hashimoto, H., Nemani, R., Hirata, R., Ichii, K., Takagi, K., Saitoh, T. M., Ohta, T., Murakami, K., Yamaguchi, Y., \& Oikawa, T. (2011). Satellite-driven estimation of terrestrial carbon flux over Far East Asia with 1-km grid resolution. Remote Sensing of Environment, 115(7), 1758-1771. http://dx.doi.org/10.1016/j.rse.2011.03.007.

Shimizu, J. Y., Ribas Júnior, U., Cancela, K. C., \& Maiochi, R. A. (2006). Cedrinho como alternativa para produção de madeira em pequenas propriedades rurais. Colombo: Embrapa.

Verrelst, J., Schaepman, M. E., Malenovský, Z., \& Clevers, J. G. P. W. (2010). Effects of woody elements on simulated canopy reflectance: implications for forest chlorophyll content retrieval. Remote Sensing of Environment, 114(3), 647-656. http://dx.doi.org/10.1016/j.rse.2009.11.004.

United States Geological Survey - USGS. (2013). Aquisição de imagens orbitais digitais gratuitas do satélite Landsat8: data de passagem 04/08/2013 EUA. Reston. Retrieved in 2018, March 22, from http://landsat.usgs.gov

Wang, W., \& Liang, S. (2008). Estimation of high-spatial resolution clear sky long wave downward and net radiation over land surfaces from MODIS data. Remote Sensing of Environment, 113(4), 745-754. http://dx.doi.org/10.1016/j.rse.2008.12.004.

Wang, Y., Woodcock, C. E., Buermann, W., Stenberg, P., Voipio, P., Smolander, H., Häme, T., Tian, Y., Hu, J., Knyazikhin, Y., \& Myneni, R. B. (2004). Evaluation of the MODIS LAl algorithm at a coniferous forest site in Finland. Remote Sensing of Environment, 91(1), 114-127. http://dx.doi.org/10.1016/j.rse.2004.02.007.

Watson, D. (1947). Comparative physiological studies on the growth of field crops: Il. The effect of varying nutrient supply on net assimilation rate and leaf area. Annals of Botany, 11(4), 375-407.

http://dx.doi.org/10.1093/oxfordjournals.aob.a083165.

Author contributions: CTP: conceptualization, data curation, formal analysis, methodology, software, writing - original draft, writing - review \& editing; MFN: formal analysis, investigation, project administration, resources, supervision; LRT: data curation, project administration, validation, visualization, writing - original draft, writing - review \& editing; MDJr: investigation, project administration, supervision 